\title{
Triclosan-containing toothpastes reduce plaque and gingivitis
}

\author{
Is a triclosan/copolymer and fluoride dentifrice more effective at improving \\ plaque control and gingival health than a fluoride dentifrice or no \\ intervention?
}

\begin{abstract}
Davies RM, Ellwood RP, Davies GM. The effectiveness of a toothpaste containing triclosan and polyvinyl-methyl ether maleic acid copolymer in improving plaque control and gingival health: a systematic review. J Clin Periodontol 2004; 31:1029-1033
\end{abstract}

Data sources Sources of studies were the Cochrane Controlled Trials Register, Medline and Embase. Personal files and the reference lists of all articles were checked for further studies.

Study selection Trials were selected if they met the following criteria: there had been random allocation of participants; participants were adults with plaque and gingivitis; there was unsupervised use of dentifrices for at least 6 months; and primary outcomes were plaque and gingivitis after 6 months.

Data extraction and synthesis Data were extracted by two reviewers independently. For each plaque and gingivitis index, the mean differences for each study were pooled as weighted mean differences (WMD) with the appropriate $95 \%$ confidence intervals (CI) using the random effects model.

Results Sixteen trials provided data for the meta-analysis. The triclosan/copolymer dentifrice significantly improved plaque control compared with a fluoride dentifrice, with a WMD of $-0.48(95 \% \mathrm{Cl}$, -0.64 to -0.32$)$ for the Quigley-Hein index and WMD of -0.15 (95\% $\mathrm{Cl},-0.20$ to -0.09 ) for the plaque severity index. When compared with a fluoride dentifrice, the triclosan/copolymer dentifrice significantly reduced gingivitis with WMD of $-0.26(95 \% \mathrm{Cl},-0.34$ to -0.18$)$ and $-0.12(95 \% \mathrm{Cl},-0.17$ to -0.08$)$ for the Loe and Silness index and for gingivitis.

Conclusions This systematic review indicates that a dentifrice that contains triclosan/copolymer provides a more effective level of plaque control and periodontal health than a conventional fluoride dentifrice.

\section{Commentary}

This systematic review supports, very clearly, the benefit of triclosan-containing toothpaste for reduction of plaques and gingivitis. Further, this finding was consistent in the 16 trials carried out in 7 countries that were included in the review.

The studies evaluated unsupervised brushing and thus the results should reflect what happens in every day usage by untrained patients. That said, the magnitude of the clinical impact is difficult to determine as reported in the manuscript. Data are reported as the WMD which, although a useful and meaningful measure for statisticians who must summarise data, is meaningless for a clinician or a patient. A translation of this into lay language would assist the reader in the clinical application of this information.

The authors do attempt to provide a clinical translation of the results by providing the proportion of sites with plaque and bleeding reduction: for sites with plaque the reduction was from $31 \%$ down to $16 \%$; the reduction for bleeding sites was from $24 \%$ down to $12 \%$. Both of these are, "...49\% reduction[s]." A more understandable measure would be an absolute reduction of $15 \%$ (31 to $16 \%$ ) for plaque and an absolute reduction of $12 \%$ (24 to $12 \%$ ) for bleeding.

Using absolute reduction allows one to calculate a numberneeded-to-treat [NNT; NNT $=(1 /$ absolute reduction $) 100]$. This yields an NNT of 7 for plaque reduction and an NNT of 8 for bleeding. In other words, for every 7 sites with plaque that are brushed with triclosan-containing toothpaste one would be significantly reduced, compared with the use of a non-triclosan toothpaste. Similarly, for every 8 sites brushed with triclosan, one would be significantly improved compared with a non-triclosan toothpaste.

In summary, there is a statistical and clinical benefit in triclosancontaining toothpaste. This benefit would be very difficult, however, for either a patient or a clinician to identify. The remaining significant question is: do the benefits in plaque and bleeding reduction lead to a reduction in caries or periodontal disease?

\section{Practice point}

- There is both a statistical and clinical benefit of triclosancontaining toothpaste in reduction of plaque and gingivitis.

\section{Richard Niederman}

DSM-Forsyth Center for Evidence-Based Dentistry, The Forsyth Institute, 140 The Fenway, Boston MA 02115, USA

Evidence-Based Dentistry (2005) 6, 33.

doi: $10.1038 /$ sj.ebd. 6400318 